\title{
Biosensor and its Application in Food and Dairy Industry: A Review
}

\author{
B.D. Meshram ${ }^{1 *}$, A.K. Agrawal ${ }^{2}$, Shaikh Adil ${ }^{1}$, Suvartan Ranvir ${ }^{3}$ and K.K. Sande ${ }^{2}$ \\ ${ }^{1}$ Department of Dairy Technology, College of Dairy Technology, Warud (Pusad) - 445204, \\ Maharashtra Animal and Fishery Sciences University, Nagpur, India \\ ${ }^{2}$ Department of Dairy Technology, College of Dairy Science and \\ Food Technology, Raipur (C.G.), India \\ ${ }^{3}$ Dairy Chemistry Division, National Dairy Research Institute, Karnal, \\ Haryana-132001, India \\ *Corresponding author
}

\section{A B S T R A C T}

\begin{tabular}{|c|}
\hline Keywords \\
\hline $\begin{array}{l}\text { Food and dairy } \\
\text { industry, Biosensor }\end{array}$ \\
\hline Article Info \\
\hline $\begin{array}{l}\text { Accepted: } \\
\text { 26 January } 2018 \\
\text { Available Online: } \\
\text { 10 February } 2018\end{array}$ \\
\hline
\end{tabular}

Food producers are increasingly asking for efficient quality control methods to satisfy the consumer and regulatory requirements to improve the production feasibility, quality sorting, automation and reduction in production cost and time. Generally in the food and the agricultural industries, chemical and microbiological analyses are done periodically by trained operators, which are expensive and require steps of extraction or sample pretreatment, increasing the time of analysis. Biosensors can overcome all these disadvantages by offering rapid, non-destructive and affordable methods for quality control. Considering all these aspects related to food and dairy industry, the development and use of biosensor has really became a boon. This paper highlights the basic concepts and applications of biosensor in food and dairy industry. Biosensors have the potential to produce an analytical revolution to resolve the challenges in the food and industries. This review gives an overview of various types of biosensors used in the food industry and outlines its future prospects.

\section{Introduction}

Human beings have at least five of these, i.e., noses, tongues, ears, eyes and skin. They represent the main types of sensor. In the laboratory, one of the best known types of sensor is the litmus paper test for acids and alkalis, which gives a qualitative indication, by means of a colour reaction, of the presence or absence of an acid. A more precise method of indicating the degree of acidity is the measurement of $\mathrm{pH}$, either by the more extended use of colour reactions in special indicator solutions, or even by simple $\mathrm{pH}$ papers. However, the best method of measuring acidity is the use of the $\mathrm{pH}$ meter, which is an electrochemical device giving an electrical response which can be read by a needle moving on a scale or on a digital readout device or input to a microprocessor.

A sensor is a device that measures a physical quantity and converts it into a signal which can be read by an observer or by an 
instrument. Sensors can divide into three types, namely (a) physical sensors for measuring distance, mass, temperature, pressure, etc. A physical sensor is a device that provides information about a physical property of the system. (b) Chemical sensors which measure chemical substances by chemical or physical responses. It is a device that transforms chemical information, ranging from the concentration of a specific sample component to total composition analysis, into an analytically useful signal. The chemical information, mentioned above, may originate from a chemical reaction of the analyte or from a physical property of the system investigated and (c) biosensors which measure chemical substances by using a biological sensing element. All of these devices have to be connected to a transducer of some sort, so that a visibly observable response occurs. Chemical sensors and biosensors are generally concerned with sensing and measuring particular chemicals which may or may not be biological themselves. We shall usually refer to such a material as the substrate, although the more general term analyteis sometimes used.

The quality of a food product is evaluated through periodic chemical and microbiological analysis. Such procedures conventionally use techniques such as, chromatography, spectrophotometry, electrophoresis, titrations and others. These methods do not allow an easily continuous monitoring because they are expensive, slow, need well trained operators and in some cases, require steps of extraction or sample pretreatment, elongating the time of analysis.

A biosensor can be defined as an integrated receptor transducer device, which is capable of providing selective quantitative or semi quantitative analytical information using a biological recognition element (IUPAC, 2000). It can also be defined as "analytical devices incorporating a biological material, a biological derived material or biomimic intimately associated with or within a physiochemical transducer or transducing Microsystems, which may be optical, electrochemical, thermometric, piezoelectric or magnetic" (Coulet, 1999).

\section{History}

In 1956, Clark published his definitive paper on the oxygen electrode

In 1962, he described how "to make electrochemical sensors more intelligent" by adding "enzyme transducers as membrane enclosed sandwiches".

In 1975, Yellow spring instrument Co., Ohio, USA produced the $1^{\text {st }}$ of many biosensor based laboratory analyser to be built by companies around the world.

Guilbault and Montalvo were the first to detail a potentiometric enzyme electrode, a urea sensor based on urease immobilised at an ammonium selective liquid membrane electrode.

Lubbers and Opitz (1975) described a fibreoptic sensor with immobilised indicator to measure carbon dioxide or oxygen.

They extended the concept to make an optical biosensor for alcohol by immobilising alcohol oxidase on the end of a fibre-optic oxygen sensor.

(Route, 1975) bacteria could be harnessed as the biological element in microbial electrodes for the measurement of alcohol.

Clemens et al., 1976 incorporated an electrochemical glucose biosensor in a bedside artificial pancreas and this was later marketed by Miles (Elkhart) as the Biostator. 
In the same year, La Roche (Switzerland) introduced the Lactate Analyser LA 640 in which the soluble mediator, hexacyanoferrate, was used to shuttle electrons from lactate dehydrogenase to an electrode.

In vivo application of glucose biosensors was reported by (Shichiri et al., 1982) who described the first needle-type enzyme electrode for subcutaneous implantation.

In 1987, a pen-sized meter for home bloodglucose monitoring formed the basis for the screen-printed enzyme electrodes launched by MediSense (Cambridge, USA).

The electronics have since been redesigned into popular credit-card and computer-mouse style formats, and MediSense's sales showed exponential growth, reaching $\$ 175$ million per annum by 1996, when they were purchased by Abbott

The idea of building direct immunosensors by fixing antibodies to a piezoelectric or potentiometric transducer had been explored since the early seventies. The BIAcore (Pharmacia, Sweden) produced commercial model of this technology in 1990.

\section{Basic concepts}

A biosensor consists of a bio-element and a sensor-element. The bio-element may be an enzyme, antibody, living cells, tissue, etc., and the sensing element may be electric current, electric potential, and so on. Different combinations of bio-elements and sensorelements constitute several types of biosensors to suit a vast pool of applications.

The bioreceptor is a biomolecule that recognizes the target analyte whereas the transducer converts the recognition event into a measurable signal. The uniqueness of a biosensor is that the two components are integrated into one single sensor. This combination enables one to measure the target analyte without using reagents. For example, the glucose concentration in a blood sample can be measured directly by a biosensor (which is made specifically for glucose measurement) by simply dipping the sensor in the sample. This is in contrast to the conventional assay in which many steps are used and each step may require a reagent to treat the sample. The simplicity and the speed of measurement are the main advantages of a biosensor.

The "bio" and the "sensor" elements can be coupled together in one of the four possible ways: Membrane Entrapment, Physical Adsorption, Matrix Entrapment, and Covalent Bonding. In the membrane entrapment scheme, a semi permeable membrane separates theanalyte and the bio-element, and the sensor is attached to the bio-element.

The physical adsorption scheme is dependent on a combination of Van der Waals forces, hydrophobic forces, hydrogen bonds, and ionic forces to attach the biomaterial to the surface of the sensor. The porous entrapment scheme is based on forming a porous encapsulation matrix around the biological material that helps in binding it to the sensor. In the case of the covalent bonding the sensor surface is treated as a reactive group to which the biological materials can bind.

\section{Generations of biosensors}

Biosensors are divided into 3 generation depending upon their integration level (Fig. 1).

\section{First generation}

In the simplest approach the biocatalyst is entrapped between or bound to membranes and this arrangement is fixed upon the surface of the transducer. 


\section{Second generation}

The immediate adsorptive or covalent fixation of the biologically active component to the transducer's surface permits the elimination of the semi permeable membrane.

\section{Third generation}

The direct binding of the biocatalyst to an electronic device that transducers and amplifies the signal, e.g., the gate of a field effect transistor, is the basis for a further miniaturization of biosensors.

\section{Prerequisites for a biosensor}

While developing a biosensor system following requirements are very essential and considered for successful and commercially viable project. a. Selectivity: The biosensor device should be highly selective for the target analyte and show minimum or no cross reactivity with moieties having similar chemical structure.

Stability and operating life: As such most of the biological compounds are unstable in different biochemical and environmental conditions.

The biological element used should be interfaced such that the activity is retained for a long time so as to make the device marketable and practically useful in the field

Sensitivity: The biosensor device should be able to measure in the range of interest for a given target analyte with minimum additional steps such as pre cleaning and pre concentration of the samples.

Linearity of response: The linear response range of the system should cover the concentration range over which the target analyte is to be measured.
Reproducibility of signal response: When samples having same concentrations are analyzed several times, they should give same response.

Quick response time and recovery time: The biosensor device response should be quick enough so that realtime monitoring of the target analyte can be done efficiently. The recovery time should be small for reusability of the biosensor system.

\section{Principle of biosensors}

The basic principle of biosensor technology is to convert a biologically induced recognition event (e.g., enzyme, antibody) into a detectable signal, via a transducer and processor. The end result is a display depicting both the presence and the concentration of the target analyte. The bioreceptor is a biomolecule that recognizes the target analyte 21. A bioreceptor can be a tissue, microorganism, organelle, cell, enzyme, antibody, nucleic acid and biomimic20.

The transducer converts the recognition event into a measurable signal21Transduction may be optical, electrochemical, thermometric, piezoelectric, magnetic and micromechanical or combinations of one or more of the above techniques

Biosensor $=$ Bioreceptor + Transducer

Analytical chemistry plays an important role in food quality parameters because almost every sector of industry and public service relies on quality control. A food quality biosensor is a device, which can respond to some property or properties of food and transform the response(s) into a detectable signal, often an electric signal. This signal may provide direct information about the quality factor(s) to be measured or may have a known relation to the quality factor. 
The main methods of immobilization, particularly for enzymes, include physical adsorption, entrapment in a matrix (using gels, polymers, or printing inks), covalent binding, or electrochemical polymerization and photo polymerization. Physical adsorption is generally based on interactions such as van der Waals forces between the biological element and the transducer.

\section{Components of biosensors}

\section{Transducers}

A transducer is a device that is activated by energy from one system and supplies energy, often in another form, to a second system. In biosensors this means that energy produced directly or indirectly by a biological reaction is generally converted into an electrical signal. The four main types of transducers used in biosensors are electrochemical, optical, piezoelectric and thermal transducers. Electro chemiluminescence devices are often considered optical, even when the excitation requires electrical energy. The biological element can be very important for the right choice of transducer. Enzymatic reactions are easily monitored, electrochemically or thermometrically, whereas mass sensitive devices are usually not used for enzymatic biosensors. On the other hand, piezoelectric sensors can detect affinity reactions of antigen and antibody or DNA without a label. Other transducers frequently require some sort of label for affinity measurements (Leonard et al., 2003).

\section{Electrochemical transducers}

Various electrochemical techniques have been employed in biosensors. These methods include amperometry, potentiometry, impedance and conductivity methods. The most popular technique is amperometry followed by potentiometry.

\section{Amperometric transducer}

Amperometric biosensors are based on the measurement of a steady state current produced when a constant potential is applied. This current can be related to an electrochemical species that is consumed or produced by the biological element. The electrochemical set-up frequently consists of a working electrode such as gold, platinum, glassy carbon, graphite or carbon paste, a reference electrode such as $\mathrm{Ag} / \mathrm{AgCl}$ and an auxiliary electrode, often made from carbon or platinum. The constant potential is applied by a potentiostat.

Amperometry is probably the most common detection method in biosensors due to its simplicity and sensitivity. The biological element can be directly immobilized on the electrode and is very often an enzyme. During the enzymatic reaction, an electrochemically active species can be produced or consumed by the enzyme and this species can be oxidized or reduced at the electrode. In the ideal case, the measured current is directly proportional to the analyte concentration.

The best-known example for enzyme based biosensors is the detection of glucose with glucose oxidase. The enzymatic reaction consumes $\mathrm{O}_{2}$ and produces $\mathrm{H}_{2} \mathrm{O}_{2}$, and both can be detected amperometrically. A great amount or research has been carried out in the field of amperometric enzyme biosensors. More recent biosensors use mediators for the electron transfer or enzymes with a direct electron transfer from the active centre to the electrode (Glaser, 2000).

\section{Potentiometric transducers}

Potentiometric biosensors measure potential differences under zero current conditions. Antibody-antigen binding induces a small change in the charge of the proteins that can 
be potentiometrically detected and since this charge is very small, the method is not very sensitive.

Ion selective electrodes (ISEs) are based on potentiometric measurements and can be used for mainly enzyme based biosensors. The change in $\mathrm{pH}$ due to enzyme activity for example can easily be monitored with a $\mathrm{pH}$ sensitive ISE. The potential that develops across an ion selective membrane is measured. The first potentiometric biosensor was developed by Guilbault and Montalvo for urea in 1969. More recent potentiometric devices are based on field-effect transistor (FET) devices (Mello and Kubota, 2007).

\section{Optical transducers}

Optical sensing is a fast growing analytical discipline not only in the area of biosensors, but sensors in general. Optodes (or optrodes) can employ many different optical phenomenons such as absorbance, fluorescence, phosphorescence, polarization chemiluminescence, bioluminescence and surface plasmon resonance (SPR). Furthermore many of these techniques can be used in various modes such as intensity or decay time.

The growing market for fiber optic devices is very beneficial for the analytical scientist. The development in this market is accelerated by the interest of telecommunication companies in optoelectronics. The capital invested by these companies in fiber optic research allows developments much faster than usual. Another important development for optical sensing is the availability of inexpensive, but stable and accurate light sources. Whereas, older instruments had to be based on expensive and often big light sources, the development of light emitting diodes (LEDs) allows the integration of these small and often cheap devices into sensors. A few years back, the market for LEDs was restricted to only a few wavelengths, but intense research has made LEDs available in many wavelengths. Mass production or fibers for phone lines and laser diodes for CD players has reduced the cost of such devices drastically and improved the state of art of developing and producing these units, also with regard to sensor specific devices (Girta, 1997).

\section{Surface Plasmon Resonance (SPR)}

SPR occurs when light is internally reflected at the interface of a material with high refractive index and a material with low refractive index. Between these two layers, a thin layer of a good conductor such as gold or silver is required (Glaser, 2000) an EW developed at this interface can interact with electron packages in the conductive layer. A very specific energy is required to raise these so called surface plasmons. The plasmon excitation energy is lost from the reflected light and can be measured with monochromatic light that is reflected at different angles. A minimum in reflectance at a certain angle (resonance angle) indicates excitation of plasmons (fig. 5).

The evanescent waves (EW) and the energy required to raise the surface plasmons are dependent on the refractive index of the medium with the low refractive index. Molecules binding to the surface of the interface layer influence the refractive index within the penetration depth of the electromagnetic wave. Therefore, antibodyantigen interactions or Deoxyribonucleic acid (DNA) binding can be monitored label-free with SPR. SPR is a charge density oscillation and associated with a surface plasma wave (SPW). This wave penetrates both, the optical dense and less dense medium. In the case of gold, the SPW can be excited by optical waves, such as EWs. The SPW is around ten times more intense than the EW and very 
sensitive to changes in refractive index ortho dielectric layer (Homola and Cauglitz, 1999).

SPR can not only be measured by the resonance angle, but also by intensity and a number of other techniques, however in biosensor applications the angular method is certainly the most popular one. The technique not only allows the label- free detection of bio-affinity reactions, but in most cases does not require washing steps. In 1990, the first SPR based biosensor was commercially available (BlAcore) and was constantly developed and updated. Other companies such as Quantech and Texas Instruments followed with SPR biosensor devices.

\section{Piezoelectric transducers}

The phenomenon of piezoelectricity is used for mass sensitive transducers (Luong and Guilbult, 1999).

The material mostly used for piezoelectric biosensors is quartz, cut at a special angle (e.g. AT-cut). The transducer is therefore, often referred to as quartz crystal microbalance (QCM).

If an oscillating electric field is applied across the quartz disc with two deposited electrodes, an acoustic wave is induced that propagates through the crystal. The frequency depends in principle on the crystal properties such as density, chemical structure and the orientation the crystal is cut. The frequency is also influenced by mass deposited onto the crystal surface (or in many cases onto the electrode surface which is deposited on the crystal). This allows very sensitive detection of small mass changes on the crystal surface (fig 6). AT-cut crystals are often used, since their temperature coefficient is zero and they only vibrate in their thickness shear mode, reducing interferences that could be caused by other vibration moles.
In principle, two waveforms are used for piezoelectric biosensors. One is a surface acoustic wave (SAW) device. The two electrodes are placed on one side of the crystal and a standing surface wave is created. High frequencies of $30-200 \mathrm{MHz}$ give the crystal a very good theoretical sensitivity, but due to practical difficulties biosensors are mainly based on bulk acoustic wave (BAW) devices (Leonard et al., 2003).

\section{Thermometric transducers}

Thermometric biosensors exploit the change of heat absorption or evolution that occurs during biochemical reactions. This heat fluctuation is reflected in a change of temperature within the reaction medium. Sensitive thermistors are used to monitor the temperature change. Most biological reactions are exothermic. Enzymatic reactions are associated with enthalpy changes of 20$100 \mathrm{~kJ} / \mathrm{mol}$.

Insufficient specific enthalpy changes can sometimes be amplified by consecutive reactions with higher enthalpy changes. Measurements can be improved by coimmobilizing enzymes for signal amplification or by using high-protonation enthalpy buffers such as TRIS (Giese, 2002).

\section{Bio recognition layer}

The sensing element in a biosensor is a highly specific bioreceptor. Compared to most chemical sensing elements, these bioreceptors show remarkable affinity towards one particular analyte or a family (group) of analytes. Over the years, a number of different biological elements have been used in biosensors. The most important biomolecule for biosensors are enzymes with their affinity towards certain substrates. Biorecognition between antibody and antigen, as used for immunological sensors are also important. 
Other biomolecules frequently used are DNA strands with affinity towards complementary strands or other molecules. Lecitin was used due to its affinity to sugars. Other systems include whole cells, bacteria, yeasts, subcellular fractions, membrane receptors and tissue slices.

Depending on their biological element, biosensors can be catalytic sensors or affinity sensors (Tothill, 2001). In catalytic sensors, an enzymatic reaction often involving the analyte, causes the concentration change of a detectable compound. In affinity sensors, the binding between the analyte and receptor is monitored, e.g. with antibodies or DNA.

\section{Immobilization}

The immobilization of the biological element onto the transducer is very important for the biosensor performance. The optimal immobilization method would yield a biomolecule immobilized on the surface of a transducer, retaining its full activity with longterm stability regarding its function and immobilization. Furthermore, the biomolecule should be fully accessible for substrate, analyte, coreactant, antigen, antibody or oligonucleotide. The transducer should be unaffected by the immobilization step. Many immobilization methods can fulfill a number of these requirements, but they also have disadvantages. Therefore, the immobilization method has to be chosen and adapted for the particular bio element, transducer, matrix and other assay requirements (Kuhnert et al., 2000).

The most common immobilization methods used for biosensors can be divided into physical and chemical methods. Physical methods include adsorption, entrapment, encapsulation and confining. Chemical methods are cross- linking and covalent immobilization. However, cross-linking of biomolecule is mostly carried out to improve the stability of physical methods (Leonard et al., 2003).

\section{Physical Adsorption}

Physical adsorption of biomolecules to solid surfaces is a simple technique. Many different surfaces are used for adsorbing biomolecules. These materials include cellulose, collagen, PVC, gold and carbon. Proteins are attached to these surfaces by low energy bonds such as charge-charge interactions, hydrogen bonds, Van der Waals forces and hydrophobic interactions.

The advantage of adsorption as an immobilization method is its simplicity. Frequently, the surface is only incubated in the protein solution for a certain time and then washed to remove excess protein. However, the stability of the protein layer is generally poor and can be affected by many factors such as $\mathrm{pH}$, ionic strength or temperature. To improve the stability of the adsorbed molecules, they are sometimes cross-linked with bifunctional reagents such as glutaraldehyde. This results in an adsorbed network rather than adsorbed molecules. Unfortunately, the process of cross-linking can deactivates the biomolecules to some extent (Olinda et al., 2003).

\section{Entrapment}

The biological element can be entrapped in a three dimensional polymeric lattice. This is normally achieved by forming a networked polymer gel around the biomolecule. Starch gels, nation, silicate and polyacrylamide gels are frequently used. The network can be formed by polymerising a three dimensional structure or by cross-linking two-dimensional polymer strands. Polymerisation is also carried out electrochemically. Pyrrole for example forms a conducting layer (poly-pyrrole) on the 
electrode surface and allows the entrapment of enzymes. Whereas conducting polymers allow polymer films of any thickness, the formation of non-conducting polymers (e.g. poly-phenol) is restricted to thin compact films which growth is self- limited. The gel can also be formed with a technique called co-reticulation. A mixture of excess of inactive protein and the active biomolecule are cross- linked. The resulting network is a gel, formed by the cross-linked inactive protein with the active biomolecule trapped and cross-linked in the gel (Evangelyn et al., 2003).

\section{Encapsulation and Confining}

Encapsulation and confining as an immobilization method is mainly used for enzymes. A simple system that was employed in early biosensors is the retention of enzymes on transducers, such as electrodes, by semipermeable membranes, such as dialysis membranes. Substrates and products can cross this barrier, but the enzyme cannot.

Biomolecules can also be confined in microcapsules of either semi-permeable materials or liposomes. However, even when the enzymes are immobilized in the microcapsules, association with the transducer is often difficult (Mello and Kubota, 2007).

\section{Covalent binding}

The immobilization of biomolecules on solid supports by covalent coupling usually leads to stable linkages. Proteins can be bound to an activated surface via their amino acid residues or terminal groups. These groups include amino, thiol, carboxyl, phenolic, imidazole, disulfide, hydroxyl and thioether groups.

An important factor for covalent immobilization is the support material and especially the presence of functional groups. These functional groups can be present either directly at the solid support or be introduced via another matrix, such as membranes.

If an additional matrix is used to introduce functional groups to the solid support, the range of these groups is very broad. Polyamide, polysaccharide, polyvinyl type membranes, amongst many others, are derivative to contain hydroxy, aldehyde, amine, carboxyl, thiol or other residues. Preactivated membranes are commercially available. The reaction between membrane and protein sometimes involves chemical activation and mild chemistry, but often it is only required to dip the pre-activated membrane into a protein solution or apply a drop of this solution onto the membrane. The variety and availability of these immobilization procedures allow the comparison of different membranes for proteins. Every protein is different and has different amino acid residues exposed. Membranes exhibiting excellent re suits with one protein may be unsuitable for another (Mello and Kubota, 2002).

\section{Applications of biosensors in food industries}

\section{Biosensors in food quality}

It is obvious that many batch operations in the food industry are being replaced by continuous processing and high degree of automation. Accordingly, there is an increasing demand for instruments suitable for automatic quality control through the process and at the end of the line so that the real time state of the process can be described.

Biosensors obviously offer food industry monitoring of specific analyte at real-time and a feedback control. This will not only increase the food safety but also provide less effective control, less employment, time and energy saving (Velasco-Garcia and Mottram, 2003). 
Biosensors can be used as analytical tools in some food industries, especially applied to the determination of the composition, degree of contamination of raw materials and processed foods, and for the on-line control of the fermentation process. Despite the enormous diversity of research involving biosensors for the food industry, its application in this area for any analyte is still restricted (Mello and Kubota, 2002).

Some important applications of biosensors to the area of food quality control are as follows

\section{Detection of microorganisms}

Conventional methods to determine and specify microorganisms are time consuming and laborious. They are based on so-called colony counts on solid media and often include different enrichment and isolation steps on selective media. The confirmation of the identity of the isolated microorganism is achieved by microscope, biochemical and immunological characteristics. This leads to total detection times of several days which are the major disadvantage of conventional plating methods. However, improved analytical methods have been developed which predominantly use the advantages supplied by immunological or t-based methods for the past decade biosensors have become more and more important for the determination of microorganism. Very specific antibodies can be produced against surface antigens of various microorganisms. In this way, an immunosensor can discriminate between different organisms. In combination with different transducers (e.g. piezoelectric materials or optical fibers) antibodies have been successfully employed for the detection of microorganisms. Most applications focus on confirning the absence of pathogenic organism like Salmonella species and Escherichia coli species. It is worth to mention that many strains of Escherichia coli are known to be dangerous human pathogens that can cause life-threatening conditions including bloody diarrhea, hemorrhagic colitis, renal failure and meningitis (Kuhnert et al., 2000). In recent years, various types of biosensors have been developed which could help in overall quality control in food processing plants by detecting pathogens within minutes of sampling. If pathogens are found with on- or near-line bios sensors, then food processors can make decisions more quickly about applying treatments, minimizing the chance of a contaminated final product (Velasco-Garcia and Mottram, 2003).

\section{Quality control of modified atmosphere packages}

Improper package design or temperature abuse during handling may cause fruits and vegetables in modified atmosphere packages to be exposed to low, injurious $\mathrm{O}_{2}$ levels associated with the production of fermentation volatiles quality loss and eventually product breakdown (Velasco-Garcia and Mottram, 2003). Excessively low package $\mathrm{O}_{2}$ also may promote growth of dangerous pathogens (e.g. Clostridium botulinum).

The detection of ethanol would provide a sensitive technique for low- $\mathrm{O}_{2}$ injury identification. A commercial ethanol biosensor composed of a chromagen and immobilized enzyme: alcohol oxidase and peroxidase have been tested. Alcohol oxidase catalyses oxidation of ethanol into acetaldehyde and $\mathrm{H}_{2} \mathrm{O}_{2}$ in the presence of $\mathrm{O}_{2}$ and peroxidase (an $\mathrm{H}_{2} \mathrm{O}_{2}$ decomposing enzyme) catalyses oxidation of the chromagen causing a colour change. The biosensor detects ethanol to levels below the human olfactory threshold $(10 \mu 1 / 1)$ ethanol in gas phase at $50^{\circ} \mathrm{Cwith}$ a $15 \mathrm{~s}$ exposure. The onset of low $\mathrm{O}_{2}$ injury was detected in lightly processed lettuce, cauliflower, broccoli und cabbage modified atmosphere packages as 
measured by accumulation of headspace ethanol (Smyth et al., 1996). The response of the biosensor was very similar to the one measured by gas chromatography, which is expensive and requires technical expertise. The biosensor could also be useful to monitor ethanol dining controlled atmosphere storage of apples, rot development in stored potato tubers or any application where ethanol accumulation can be associated with a loss of quality.

\section{Fish freshness analysis}

Fish freshness has been evaluated chemically and expressed as $\mathrm{K}$-value which is useful index of raw fish grade. However, the $K$ value approach requires the sample preparation and the complicated sensor system with several kinds of biochemical substances because the $K$-value is calculated from the concentrations of inosine "5-monophosphate (IMP), inosine (HxR) and hypoxanthine (Hx) in the fish-extract solution, with several kinds of biochemical process reagents. Then, a newly approach is required at fish markets, restaurants and kitchens, i.e., non-destructive methods with simple biochemical reaction, such as smell evaluation of putrid fish-odour with higher sensitivity of human smell sense (Mitsubayashi et al., 2004).

Trimethylamine (TMA) is typical and common fish-odour substance in seafood, and is produced by the decomposition of trimethylamine N-oxide (TMAO) in sea creatures. The fact is that fresh marine products contain little TMA Mitsubayashi et al., (2004) constructed aTMA biosensors by immobilizing flavine containing mono oxygenase type 3 (FMO3), as one of drug metabolizing enzymes in human liver, onto a sensitive area of a dissolved oxygen electrode. This sensor with flow injection analysis (FIA) was calibrated against TMA solutions. It was obvious that the TMA sensor with FMO3 would be convenient device for evaluating fish-freshness (Coefficient of variation $4.39 \%$, $\mathrm{n}=5$ ). The behavior of the biosensor was evaluated using standard TMA solution as the typical putrefactive substance in fish, with a flow injection analysis system including a computer-controlled potentiostat at a fixed potential of $600 \mathrm{mV}$ versus Ag/AgClas counter/reference. The sensor output induced by FMO3 enzyme reaction was continuously monitored on a computer display and saved on the hard disk for later analysis.

\section{Quality control of meat}

Commercialized sensing instruments for quality control of meat are meat check and bio check sensors. The meat check is a four electrode array attached to a knife which can be inserted into meat to measure the glucose gradient immediately below the surface. The size of the gradient is related to microbial activity on the surface of the meat and is regarded as a sound indicator of meat quality. The device provides in seconds what laboratory-based microbiology takes days to test. The bio checks method transformed the glucose sensor into a device capable of detecting and quantifying microorganisms in aqueous solutions. The system transfers electrons from the respiratory pathways of microorganisms, and it is capable of detecting bacteria in less than two minutes (Maines et al., 1996). Table 1 shows the range of analyte monitored in meat products by biosensors.

The lactic acid concentration leads to conclusions concerning the pre -mortem metabolic situation, physical stress and deficiency in the meat quality. Bergann et al., (1999) reported on enzymatic biosensor based on immobilized lactate oxidase as bioreceptor and an amperometric transducer. The biosensor estimate lactic acid without special sample preparation, very quickly and at low cost. 
Fig.1 Generations of biosensors

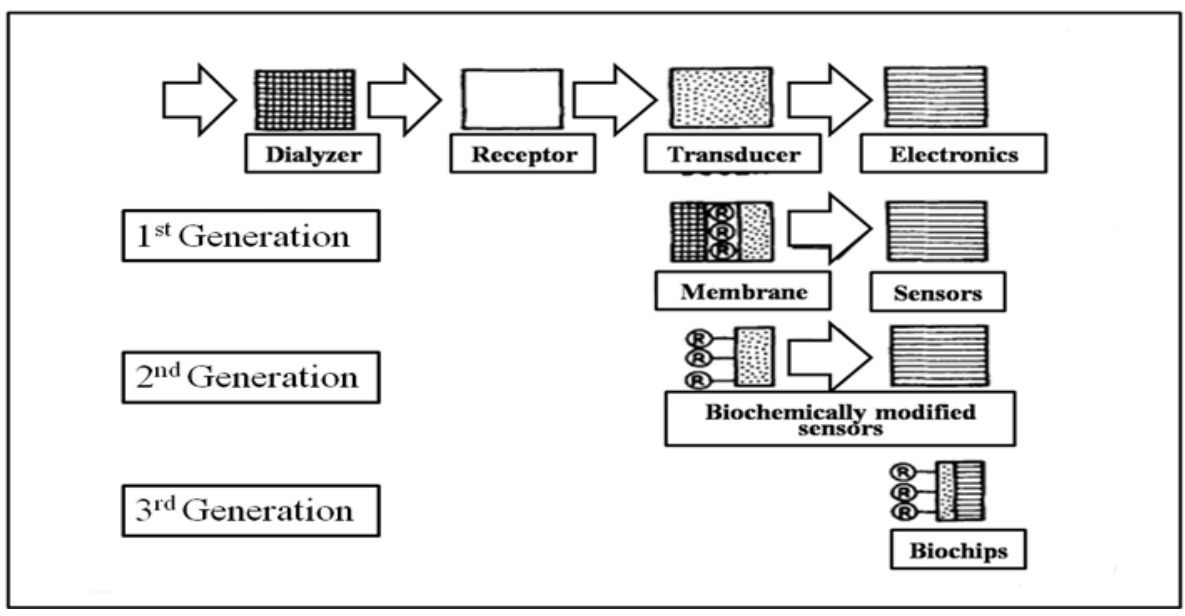

Fig.2 Schematic diagram of a biosensor

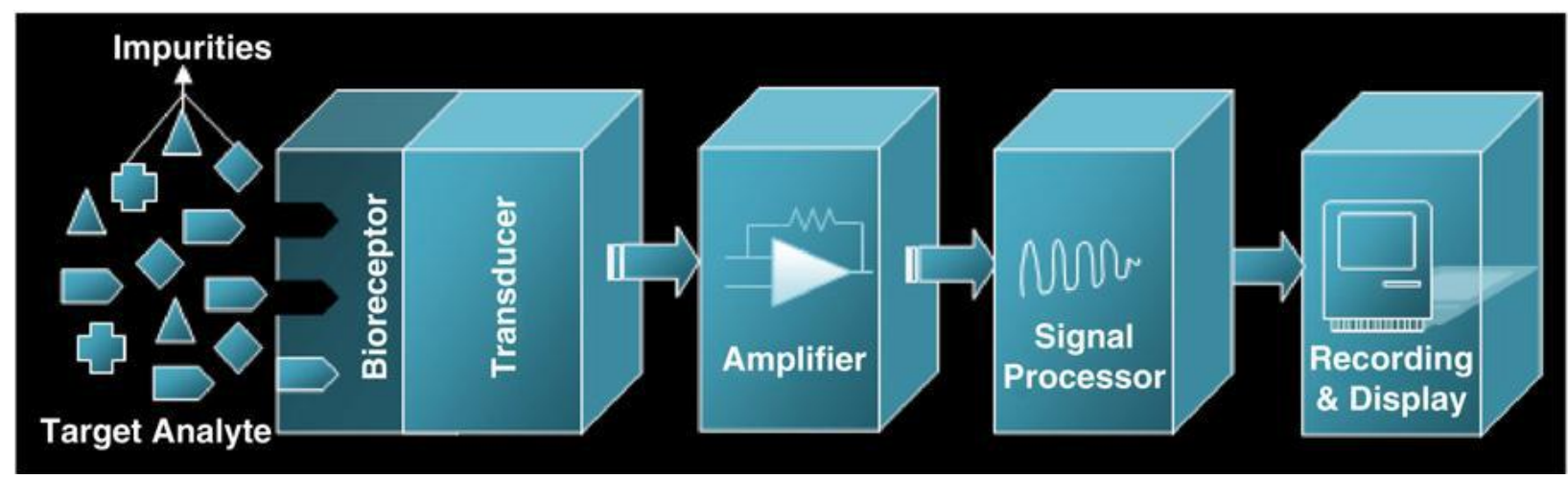

Fig.3 Principle of biosensor

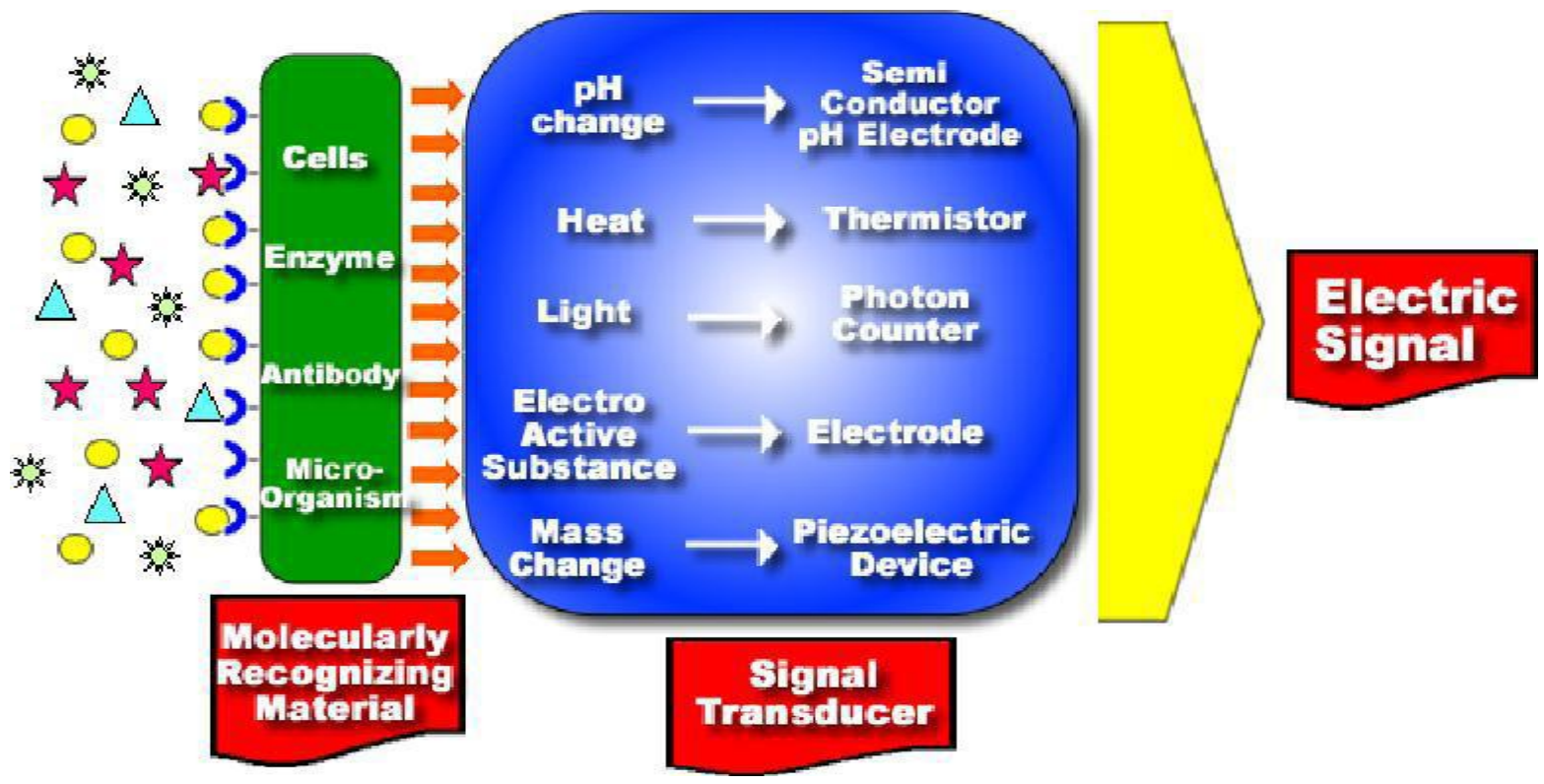


Fig.4 Schematic diagram of optoelectric sensor by using bromocresol green bioreceptor

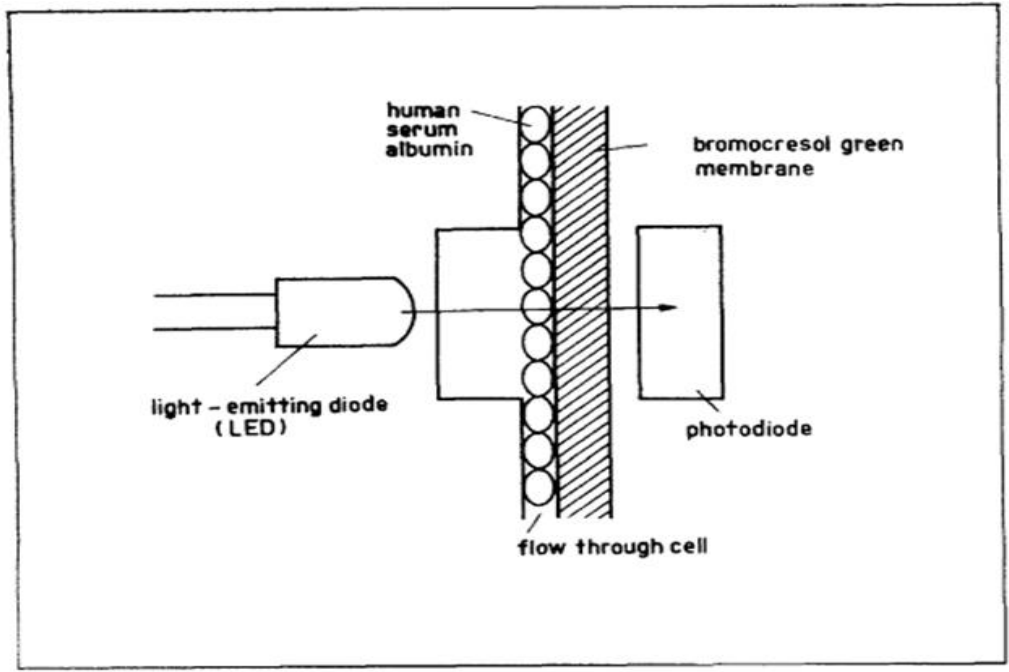

Fig.5 Surface Plasmon Resonance (SPR)

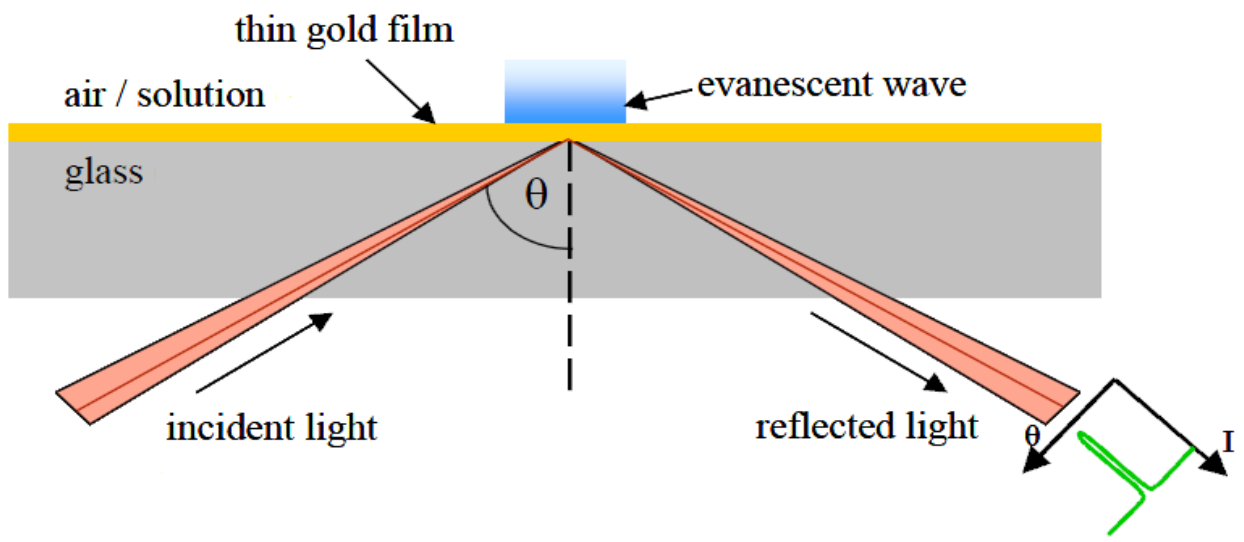

Fig.6 Piezoelectric transducer with quartz crystal

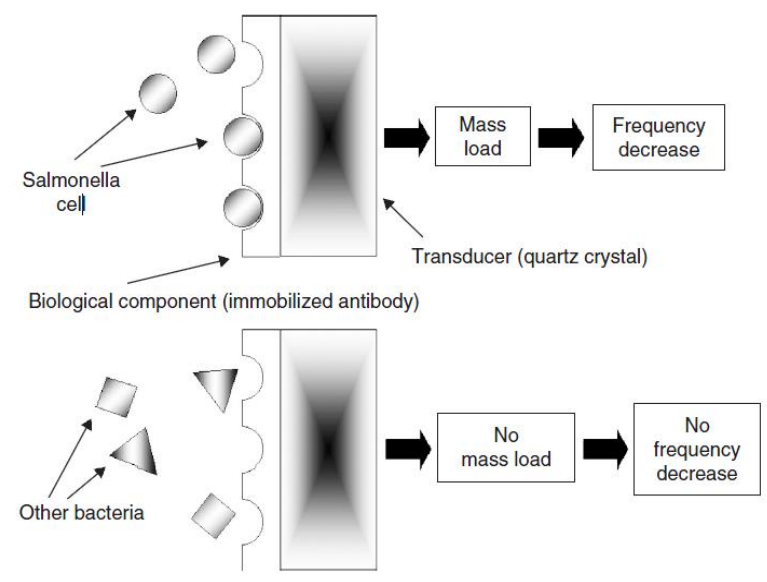


Fig.7 Tea biosensor designed in CFTRI, Mysore

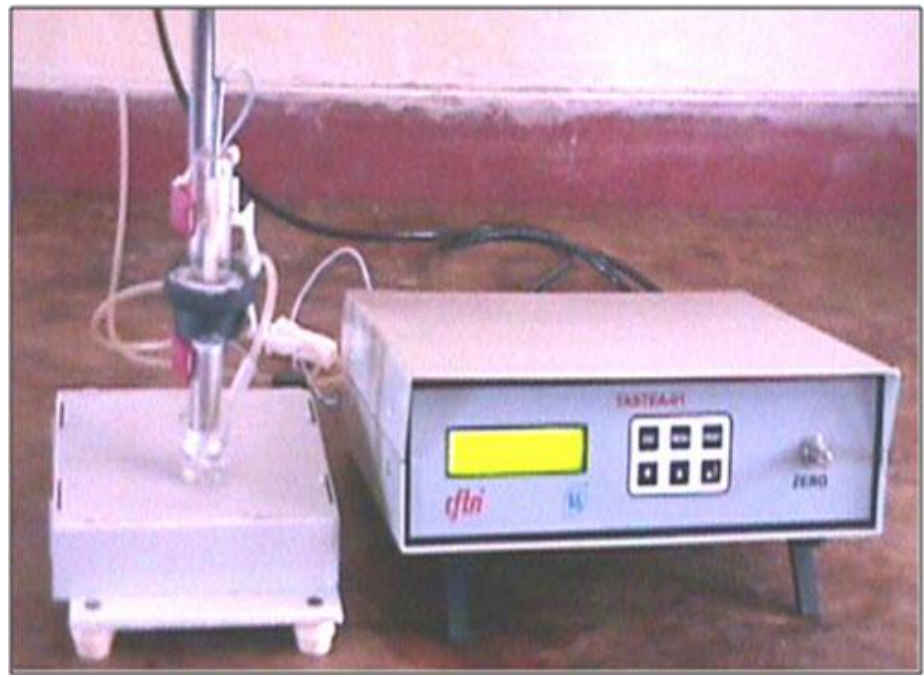

Table.1 Range of analyte monitored in meat products by biosensors

\begin{tabular}{|l|}
\multicolumn{1}{|c|}{ Analytes } \\
\hline Amines \\
\hline Amines \\
\hline Biogenic amines \\
\hline Histamine \\
\hline Hypoxanthine \\
\hline Bacteria \\
\hline Bacteria \\
\hline Bacteria, viruses, \\
spores
\end{tabular}

\begin{tabular}{|c|c|}
\hline Food matrix & Bio component \\
\hline Fish & Diamine oxidase (DAO) \\
\hline Fish freshness & Hypoxanthine oxidase \\
\hline Fish & Diamine oxidase \\
\hline Sea foods & Histamine oxidase \\
\hline Fish and its freshness & Xanthine oxidase (XOD) \\
\hline Chicken & Anti-Salmonella antibody \\
\hline Beef & Anti- Escherichia coli \\
\hline Hamburger, ham & Antibody \\
\hline
\end{tabular}

Table.2 Comparative results of ethanol determination from alcoholic beverages

\begin{tabular}{|l|c|c|c|c|}
\hline \multirow{2}{*}{ Sample } & \multicolumn{2}{|c|}{ Biosensor } & \multicolumn{2}{c|}{ Spectrometric method } \\
\hline & Conc.(M) & Conc. \% vol & Conc.(M) & Conc. \% vol \\
\hline Beer 1 & 0.87 & 4.00 & 0.88 & 4.05 \\
\hline Beer 2 & 1.95 & 8.90 & 2.01 & 9.25 \\
\hline Black beer & 2.17 & 10.00 & 2.08 & 9.57 \\
\hline Vodka & 6.95 & 31.90 & 7.06 & 32.50 \\
\hline Cognac & 8.69 & 40.00 & 8.81 & 40.50 \\
\hline Palinca 1 & 10.86 & 50.00 & 10.60 & 48.70 \\
\hline Palinca 2 & 13.00 & 59.80 & 13.24 & 60.90 \\
\hline
\end{tabular}


Table.3 Range of analyte monitored in milk and milk products by biosensors

\begin{tabular}{|l|l|l|}
\multicolumn{1}{|c|}{ Analyte } & \multicolumn{1}{|c|}{ Food matrix } & \multicolumn{1}{c|}{ Bio component } \\
\hline Glucose & Milk & Glucose oxidase \\
Goghurt & Milk & D-fructosidase dehydrogenase \\
\hline Fructose & Milk & Galactosidase, lactozym and Saccharomyces \\
\hline Lactose & Milk & D-fructose dehydrogenase, glucose oxidase \\
\hline Laculose & Infant formula and milk & Anti- biotin antibody \\
\hline Biotin & Infant formula and milk & Anti folic acid antibody \\
\hline Folate & Milk & Lyase oxidase \\
\hline L- lysine & Milk & Antibodies \\
\hline Antibiotics & Milk & Chlinesterase \\
\hline Pesticides & Butter, cream & Cholesterol oxidase or Horseradish peroxidase \\
\hline Cholesterol & &
\end{tabular}

\section{Monitoring of wine quality}

Wine is a complex mixture of several hundred compounds, present simultaneously, at different concentrations. The dominants ones are water, ethanol, glycerol, sugars, organic acids and various ions. Except ethanol and glycerol, other aliphatic and aromatic alcohols, amino acids and phenolic compounds are present at much lower concentrations.

Three different PQQ-dehydrogenases [glucose dehydrogenase (GDH), alcohol dehydrogenase (ADH), and glycerol dehydrogenase, (GIDH) newly isolated and purified from Erwinia spp. or Gluconobacter spp. have been used for development of biosensors for determination of key compounds in wine.

The main enzyme substrate (glucose for GDH, glycerol for GIDH and ethanol for $\mathrm{ADH}$ ) is firstly oxidized while the enzyme's cofactor is simultaneously reduced. The active form of the enzyme is regenerated via the interaction with the electrochemical mediator (Os modified redox polymer), which is maintained in its oxidized form by the positive potential applied at the electrode.

\section{Ethanol determination in alcoholic beverages}

Microbial biosensor was used to determine ethanol concentration in alcoholic beverages. Different dilutions between 40 and 500 times were performed. Results were compared with the enzymatic spectrometric method (Table 2).

The correlation coefficient of experimental data of 0.9983 shows a good correlation between biosensor and spectrometric method. The variation coefficient was no more than $5 \%$ for biosensor and $2.5 \%$ for spectrometric method, for determination realised in the same day.

\section{Free radicals and antioxidants}

Antioxidants are one of the main ingredients that protect food attributes by preventing oxidation that occurs during processing, distribution and end preparation of food. Different types of antioxidants are added to food products to increase the attributes of food product. Amperometric biosensors are generally preferred for the determination of antioxidants in various food products (Mello and Kubota, 2007). 


\section{Fresh fruits and vegetables}

The fresh produce industry illustrates many of the problems encountered across the whole food industry in terms of still generally employing archaic QC methodologies (Manikas, 2002). For example, fresh produce quality in the intact or minimally processed/fresh-cut form is initially assessed by sight; other important quality attributes include taste, smell and texture. Each of these four quality attributes can be assessed either subjectively or objectively. Typically, fruit processors reject $10 \%$ of fruits intakes. Better selection through improved quantitative QCat low-cost will inevitably result in improved overall quality for intact and minimally processed fruit products. Improved QC will probably, in the short-term, lead to increased rejections and reduce the number of concessions for fruit processors. A concession is where fruit material can be used but is expected to incur a cost penalty due to eater QC costs. Improved QC may result in a ca. $25 \%$ saving on concessions for fruit processors (Cockerill, M. Orchard House Foods, United Kingdom, Personal communication, 2004).

In reality, current standard product-orientated QC operations are inadequate and consider only appearance (e.g., color. size. and shape), presence/absence of disease, and the concentration of total soluble solids (TSS). TSS is commonly expressed as ${ }^{\circ} \mathrm{Brix}$ and is typically still measured using a hand-held refractometer. There is often poor correlation between TSS and total sugar concentration. Fruit sugars are one of the main soluble components in fresh produce that are important for flavor. In addition to sugar composition, fruit acid concentration can affect flavor directly and can regulate cellular $\mathrm{pH}$, influencing the appearance of fruit pigments within the tissue dining processing. The total titratable acidity (TTA) of fruit is not routinely measured as part of the standard QC procedures that are implemented by growers, suppliers, fresh produce distribution centers and fruit processors (Manikas, 2002). Titratable acidity is a measure of the buffering capacity of the fruit and is generally expressed as a percent of the predominant organic acid. Current standard QC operations do not use TTA due to the cumbersome and time-consuming nature of titrations. Fruit sugar/acid ratios can be used as an important index of consumer acceptability and act as one determinant of overall fruit quality. However, sugar/acid ratios are not frequently assessed for all fruit types due to primitive QC instrumentation and the requirement for skilled analytical scientists.

An initial step to improving routine $\mathrm{QC}$ assessment would constitute producing a simple and low-cost alternative to refractometry and titrations so that specific sugar and organic acid ratios can be standardized for fresh produce types. Biosensors may offer the opportunity to fulfillthis niche and allow industry to adjudge fruit quality on the basis of taste (sugar: acid ratios) rather than just appearance alone. Introducing biosensor technology within the fresh produce industry may provide the ideal solution to providing improved QC, safety and traceability methodologies. It follows that biosensor applications could be extended across the whole food industry, E.g. meat, dairy foods and beverages.

\section{Tea biosensor}

India is exporting a large quantity of black tea all over the world. Tea polyphenols play a crucial role in determining quality of black and green tea. Major quality attributes such as colour and astringency are directly linked with polyphenol contents. Therefore, it is necessary to know quantity of polyphenols in tea. Also, tea polyphenols are gaining 
importance due to their strong antioxidant properties for nutrition and health. In this context CFTRI, Mysore successfully developed an enzyme based amperometric biosensor (Fig. 7) for the determination of total polyphenol content in tea infusions. Both lab and industry trials were satisfactory for tea polyphenols detection and tea biosensor technology (Sujith Kumar et al., 2011).

\section{Determination of ascorbic acid in fruit juices}

Flow injection Potentiometric system has been developed for simultaneous determination of ascorbic acid with other parameters. This system is based on the reaction of the species with ascorbate oxidase which is immobilized on alkaline glass beads using glutaraldehyde. Fall in oxygen consumption is detected by the electrode. Oxygen consumed is proportional to the ascorbic acid content of the sample (Eshkenazi et al., 2000).

\section{Alcohol biosensor}

Online measurement of ethyl alcohol or methanol during fermentation and yeast cultivation is very important to minimize product inhibition and increase yield. Many micro-organisms use alcohols as carbon source, its concentration can be determined from the respiration activity of the cells. The respiration activity is directly measured by oxygen electrode. The biosensor system consists of immobilized yeast or bacteria, a gas permeable membrane (Teflon) and an oxygen electrode. Porous membrane retaining microbial cells is fixed on the surface of Teflon membrane of the electrode. Thus, the cells are trapped between the two membranes. A gas permeable membrane is placed on the surface of electrode and covered with nylon net. These membranes are fastened with rubber -o-rings. The steady state current obtained depend on the concentration of ethanol / methanol. The response time is 10 min at 30OC (Rajasekhar et al., 2005).

Methanol $+\mathrm{O}_{2} \longrightarrow \mathrm{H}_{2} \mathrm{O}_{2}+$ Formaldehyde Ethanol $+\mathrm{O}_{2} \longrightarrow \mathrm{H}_{2} \mathrm{O}_{2}+$ Acetaldehyde

\section{Application of biosensor in dairy industries}

\section{Online monitoring of milk}

The increasing demand for on-line evaluation of milk quality directs the industry to look for practical solutions, and biosensors are a promising possibility. (Eshkenazi et al., 2000) developed a multi-enzymatic amperometric biosensor for lactose in fresh raw milk. The characteristics of the biosensor (easy operation, rapid response, long stability suggested that this method could be used as an economical, on-line lactose measurement technique in the milking parlour. Also, some biosensors were designed to determine fat in milk (Velasco- Garcia and Mottram, 2003). (Schmidt et al., 1999) reported a microbial biosensor based on thick film technology for free fatty acids. The biosensor measures the oxygen uptake by respiratory activity of the immobilized microorganisms. Oxygen was determined by electrochemical reduction. The sensor could be applied to milk samples without previous pretreatment, having a short response high sensitivity and easy handling. However, biological research is needed to determine how sensor derived information can be used to improve the product quality other than by separating the milk into sources of high and low quality. Table 3 gives details of few range of analytes monitored in milk and milk products.

\section{Biosensor for quality control in milk}

The food industry needs suitable analytical method for quality control, that is, methids that rae rapid, reliable, specific and cost 
effective as current wet chemistries and analytical practice are time consuming and may require highly skilled labour and expensive equipment. The need arises from heightened consumer concerned about food composition and safety. The study was carried out keeping in view the recently emerging concern of the presence of urea in milk, called "synthetic milk". This urea biosensor is immobolized urease yielding bacterial cell biomass Bacillus sphaericus isolated from soil and coupled to the ammonium ion selectively electrode of a potentiometric transducer. Samples of milk were collected and analyzed for the presence of urea by the developed biosensor with a response time as low as two minutes. The results were in good correlation with pure enzyme system. However, it is worth the mentioned that since milk is a complex system it contains much interference, which makes conventional methids less reliable (Verma and Singh, 2003).

\section{On- line determination of lactose concentration in milk}

The cascade enzyme biosensor is used for online determination of lactose content of milk. The enzyme galactosidase (GAL) cleaves the disaccharide lactose producing glucose and galactose. The glucose reacts with glucose oxidase (GOD) to form $\mathrm{H}_{2} \mathrm{O}_{2}$. Horseradish peroxidase (POD) oxidizes $\mathrm{H}_{2} \mathrm{O}_{2}$ with 5-ASA (aminosalicyclic acid) as a mediator. The oxidized form of the mediator is reduced at the electrode resulting in an amperometric signal proportional to the lactose concentration (Ferreira et al., 2003)

\section{Milk urea biosensor}

Protein supplements in animal feeds are costly. There is an efficient conversion of feed protein into milk protein. Excessive levels of nitrogen derived from feed may increase the systemic urea concentration of milk without increase in milk production. This high concentration of urea may impair reproductive performance and also causes excessive nitrogen in dairy waste which is environment pollution. The milk urea nitrogen (MUN) data helps in adjusting the nutritional programme of the herd. The normal range of MUN is 5-20 mg/dl (Edward, 1999).

\section{Biosensor for organic acid}

Among the organic acids present in food i.e. glutamic acid, lactic acid and ascorbic acid are of special importance. The quality of mozzarella cheese strongly depends on the acidity of the curd. To control the acidity, biosensor has been used to measure the lactic acid. The system consists of an electrochemical (flow-through flow-jet) cell assembled with platinum sensor covered with the immobilized lactate oxidase enzyme connected to an amperometer.

Lactate $+\mathrm{O}_{2} \stackrel{\text { Lactate oxidase }}{ } \stackrel{\longrightarrow}{\longrightarrow}$ Pyruvate
$+\mathrm{H}_{2} \mathrm{O}_{2}$

The amount of lactate in the curd is detected by $\mathrm{H} 2 \mathrm{O} 2$ probe. The real time analysis of lactate allows the control of the curd ripening evaluation at different pasteurization temperature. This method is more sensitive than $\mathrm{pH}$ probe (Rajasekhar et al., 2005).

\section{Future R and D needs}

Multifunctional and versatile biosensing systems are required for analysis of multiple analytes using a single device. At the same time, reduction in physical size of the device and multi-array analysis are preferred without compromising the specificity and sensitivity of the device. There is a growing demand of lab-on-chip interfacing with biosensor systems. Novel biosensing materials aimed at 
high sensitivity, selectivity, stable and low material synthesis cost will boost the market for biosensors and also their applications in different areas. Handling of biosensors should be made simple so that even a common man can use it without the help of qualified persons. Biosensor research should be encouraged with the allotment of funds and good infrastructure for research groups in order to advance in the biosensing field.

The potential for biosensor technology is enormous and is likely to revolutionize analysis and control of biological systems. It is possible therefore to identify very different analytical requirements and biosensor developments must be viewed under this constraint. It is often tempting to expect a single sensor targeted at a particular analyte, to be equally applicable to on-line closed-loop operation in a fermenter and pin-prick blood samples. In practice, however, the parallel development of several types of sensor, frequently employing very different measurement parameters is a more realistic.

\section{References}

Bergann T., Gifley K. and Abel P., 1999. Concentration of lactic acid in carcasses and fresh meat estimation with an enzymatic-biosensors or measuring system. Fleischwirtschaff, 79: 84- 487.

Clemens AH, Chang PH, Myers RW. De Diabetologie de L'Hotel-Dieu, ProcJournees Ann, Paris, 1976.

Coulet P.M. and Blum L.J., 1999. Biosensor Principles and Applications. Marcel Dekker, New York.

Edward, J.D. (1999). Report on investigators aim to improve milk composition for increased utilization. Summer dairy dispatch, UC Davis.

Eshkenazi, Maltz E., Zio B. and J. Rishpon., 2000. Three cascaded enzymes biosensor to determine lactose concentration in raw milk. J. Dairy Sci. 83(9): 1939-1945.

Evangelyn C. Alocilja and Stephen M. Radke, 2003. Market analysis of biosensors for food safety. Biosensors and bioelectronic, 18: 841-846.

Ferreira, L.S., Souza, M.B., Trierweiler, J.O. and Hitzmann, B. (2003). Analysis of experimental biosensor of FIA lactose measurements. Brazilian J. of Chemical Engineering.20 (1): 126-133.

Giese, J. 2002. Food biosensors. Food Technology, 56(7):72-75.

Girta F., 1997. Use of on-line biosensors in food industry. J. of Qafqaz University, 1(1):138-150.

Glaser R. W., 2000. Surface Plasmon Resonance Biosensors and their applications, Kiuwer Academic/Plenum Publishers, New York.pp.195-212.

Grattan K. T. V. and T. Sun, 2000. Fiber optic sensor technology: an overview, Sensors and Actuators, 82: 40-61.

Helena S. N., Luciane D. S., Cristiano P. P. and Sandra T., 2005. Biosensor based on xanthine oxidase for monitoring Hypoxanthine in fish meat. American J. of Biochem. and Biotech, 1(2): 85-89.

Homola J., Yee S. S. and Cauglitz C., 1999. Surface Plasmon resonance sensor review. Sensors and Actuators, 54: 315.

International Union of Pure and Applied Chemistry (IUPAC), USA. 2000.

Kuhnert P., Boerlin P. and Frey J., 2000.Target genes for virulence assessment of $E$. coli isolates from water, food and the environment. FEMS Microbiol. Rev., 24: 107-117.

Lubbers, DW, Optiz, N. The $\mathrm{pCo}_{2-}$ / $\mathrm{pO}_{2}$ optode: a new probe for measurement of $\mathrm{pCo}_{2}$ or $\mathrm{pO}$ in fluids and gases. Z Res Nat C. 1975; 30:532533. 
Luong J. H. T. and Guilbault G. G., 1999.Analytical Applications of Piezoelectric Crystal Biosensor, Biosensor Principles and Applications, Marcel Dekker, New York. pp. 107138.

Maines A., Ashworth D. and Vadgama P., 1996.Enzyme electrodes for food analysis. Food Technology and Biotechnology, 34: 31- 42.

Manikas I., 2002. Evaluation of the operational performance of fresh produce distribution centers in the UK. Thesis (MScRes), Cranfield University.

Mello L.D. and Kubota L.T., 2002. Review of the use of biosensors as analytical tools in food and drink industries. Food Chemistry, 77: 237-256.

Mello LD, Kubota LT (2007) Biosensors as a tool for the antioxidant status evaluation. Talanta 72: 335-348.

Olinda F. Canhoto and NareshMagan, 2003.Potential for detection of microorganisms and heavy metals in potable water using electronic nose technology. Biosensors and bioelectronics, 5(18): 751-754.

Rajasekhar, T., Upadhaya, J.B. and Shah, B.P. (2005). Role of biosensors in dairy and food industry. Beverage and foodworld. March: 21-24.

Schichiri, M., Kawamori, R., Yamaski, R., Hakai, $\mathrm{Y}$ and Aba, H. Wearable artificial endocrime pancreas with needle type glucose sensor. Lancet, 1982; $2: 1129$ - 1131 .

Schmidt A., Standfub-Gabisch C. and Bilitewski U., 1999. Microbial biosensor for free fatty acids using an oxygen electrode based on thick film technology. Biosensors and Bioelctronics, 11: 1139-1145.

Smyth A. R., Talasila P. C. and Cameron A.C., 1996. An ethanol biosensor can detect low-oxygen injury in modified atmosphere packages of fresh-cut produce. Post-harvest Biology and Technology, 15: 127-134.

Sujith Kumar PV, Basheer S, Ravi R, Thakur MS (2011) Comparative assessment of tea quality by various analytical and sensory methods with emphasis on tea polyphenols. J Food SciTechnol 48 (4): 440-446.

Tothill I. E., 2001.Biosensors development and potential applications in the agricultural diagnosis sector. Computers and Electronics in Agriculture, 30: 205218.

Velasco-Garcia M. N and Mottram T., 2003. Biosensor technology addressing agricultural problems: A review. Biosystems Engineering, 84: 1-12.

Verma, N. and Singh, M. (2003). Disposable microbial based biosensor for quality control in milk. Biosensor amd bioelectronics; 18:1219 - 1224 .

\section{How to cite this article:}

Meshram, B.D., A.K. Agrawal, Shaikh Adil, Suvartan Ranvir and Sande, K.K. 2018. Biosensor and its Application in Food and Dairy Industry: A Review. Int.J.Curr.Microbiol.App.Sci. 7(02): 3305-3324. doi: https://doi.org/10.20546/ijcmas.2018.702.397 